\title{
Chemical Synthesis of $\beta$-(1,3)-Glucan Oligosaccharide and Its Application
}

\author{
Atsushi Miyagawa \\ Department of Life Science and Applied Chemistry, Graduate School of Engineering, Nagoya Institute of Technology, \\ Showa-ku, Nagoya, Aichi 466-8555, Japan \\ FAX: +81-52-735-5239, E-mail: miyagawa.atsushi@nitech.ac.jp
}

(Received on September 10,2017, accepted on April 2, 2018)

Key Words: $\beta$-(1,3)-glucan, $\beta$-(1,3)-glycosylation, polysaccharide, branch, immunostimulatory activity

\begin{abstract}
$\beta$-(1,3)-Glucan has been used as an anti-cancer drug owing to its immunostimulatory effects. However, the mechanism of the biological activity has yet to be elucidated. Moreover, $\beta$ - $(1,3)$-glucans used for the mechanistic studies are heterogeneous mixtures extracted from natural sources. Therefore, structure-defined $\beta$-(1,3)-glucans are required to simplify the effect caused by $\beta$-(1,3)-glucans in the study of the biological activity, and this requirement prompts the study of chemical synthesis of $\beta$-(1,3)-glucans. The glycosylation for formation of the $\beta$-(1,3)-linkage is difficult because the reactivity of the 3-hydroxy group is suppressed due to considerable steric hindrance from the protecting group at the 4-position in glucose. Moreover, introduction of protecting groups that can be deprotected selectively on the 3-position as well as the 6-position is required to synthesize $\beta$ - $(1,3)$-glucans with a branch at the 6-position, and the synthetic method should be optimized by considering the influence of the combined protective groups on the glycosylation. Recently, $\beta$-(1,3)-glucan oligosaccharide synthesis using a 4,6-O-benzylidenated glycosyl donor and acceptor has been studied strenuously, and the reactivity of the acceptor and the stereoselectivity of $\beta$-(1,3)-glycosylation were substantially improved. Consequently, linear $\beta$-(1,3)-glucan of up to 16 saccharides could be synthesized. There have been reports of a few promising examples of multiple introductions of branch residues to the linear backbone of $\beta$-(1,3)-glucan. Moreover, some applied studies using synthetic $\beta$-(1,3)-glucan oligosaccharides have already been conducted.
\end{abstract}

\section{A. Introduction}

$\beta$-Glucan (Fig. 1 (a)) is a generic term for polysaccharide chains of D-glucose linked by $\beta$-linkages (1). The $\beta$-glucans discussed here are linear and branched $\beta$-(1,3)-glucans that compose fungal cell walls. The linear $\beta$-(1,3)-glucan has $\beta$-(1,3)-linked glucose as the main chain and the branched $\beta$ - $(1,3)$-glucan has $\beta$-(1,6)-linked glucose as the branch chain on the $\beta$-(1,3)-linked main chain $(2,3) . \beta$-(1,3)-Glucans have been extracted from mushrooms and yeast. They have historically been known to possess immunostimulatory activity and have been utilized as anticancer drugs. Within the $\beta$-(1,3)-glucan family, the biological function of lentinan and schizophyllan (Fig. 1 (b)) has been studied strenuously $(4,5)$. In 2001, Brown et al. identified Dectin-1 as the $\beta$-(1,3)-glucan recognition protein and found that various lectins are involved in the immunostimulatory effect. Dectin-1 plays important roles in antitumor responses through the activation of spleen tyrosine kinase (syk) in dendritic cells (Fig. 1 (c)) (6-9).

However, the problem when studying $\beta$-(1,3)-glucan is its nature as an biological sample extracted from a natural source. It is difficult to prepare $\beta$-(1,3)-glucan polysaccharides with homogeneously branched structure in a uniform molecular weight. Therefore, detailed investigation of the biological activity of $\beta$-(1,3)-glucan such as structure-activity relationships has not been possible. This is in part due to insufficient methods for synthetic preparation of $\beta$-(1,3)-glucan with a homogeneous structure. However, by recent progress in carbohydrate chemistry such as (a)

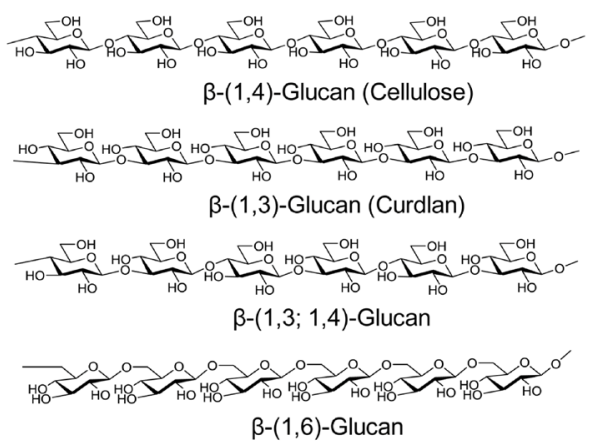

(b)
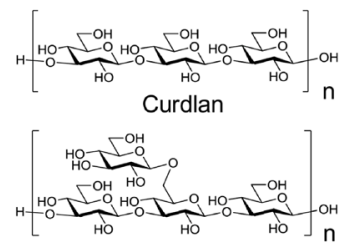

Schizophyllan

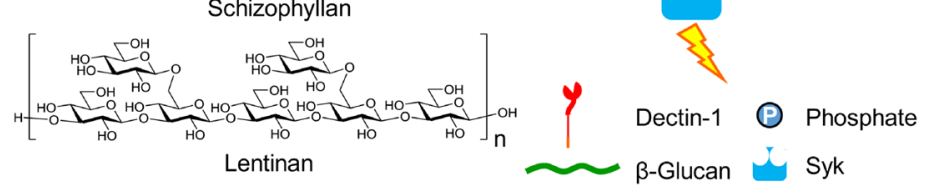

(c)

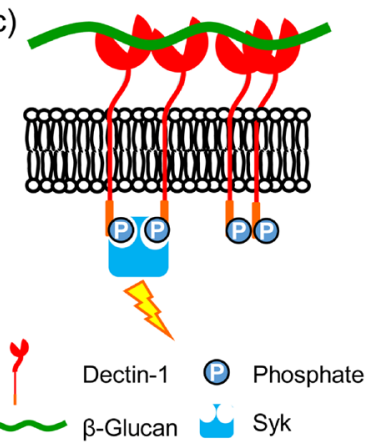

Fig. 1. (a) Structures of naturally occurring $\beta$-glucans; (b) structures of linear or branched $\beta$-(1,3)-glucans (4); (c) recognition of $\boldsymbol{\beta}$-(1,3)-glucan by Dectin-1 (9). 
varying protecting groups and implementing stereoselective glycosylation, it has become possible to synthesize $\beta$-(1,3)-glucan oligosaccharides. Additionally, the instruments for analysis have advanced, allowing glycans to be analyzed in detail regard to their structure and interaction with biomolecules. In this paper, the synthesis of $\beta$-(1,3)-glucan oligosaccharides is described; furthermore, the biological applications utilizing $\beta$-(1,3)-glucan oligosaccharides are exemplified.

\section{B. Challenge of $\boldsymbol{\beta}$-(1,3)-Glycosylation}

First, the formation of the $\beta$-(1,3)-glucoside linkage is discussed. The 2-O-acyl group on the donor is expected to direct a $\beta$-selective reaction by neighboring participation in glycosylation. However, the electron-withdrawing property of the acyl group at the 2-position result in poor electron density of the 3-OH group, which decreases the reactivity as the glycosyl acceptor (Fig. 2 (a)1). Because the 3- and 4-OH groups are situated in the equatorial direction (gauche conformation of O3-C3-C4-O4), the protecting group introduced at the 4-position hinders the glycosylation of 3-OH, decreasing the yield (Fig. 2 (a)-2). Additionally, for convergent synthesis, it is an effective to glycosylate selectively with a glycosyl acceptor having a leaving group at the 1-position. However, it was reported that aglycone transfer occurred using a thioglucoside as the glycosyl acceptor when the low reactive hydroxyl group on the glycosyl acceptor was the reaction point in glycosylation (Fig. 2 (b)) (10). In convergent synthesis of $\beta$-(1,3)-glucans, the intermediate needs to be converted to a glycosyl acceptor and glycosyl donor; therefore, the selective deprotection and activation of the anomeric position are also important points, respectively.

Next, the formation of the $\beta$ - $(1,6)$-glucoside linkage is discussed. Selective deprotection of the 3-position as well as the 6 -position is required to synthesize $\beta$-(1,3)-glucans with a branch at the 6-position. In particular, introduction of $\beta$ - $(1,6)$-branches is not necessary for all glucose residues in a main chain for construction of natural branched $\beta$-(1,3)-glucans. Therefore, glucose residues with an alternative protecting group that can be selectively removed should be used as the branching glucose residues. The synthesis step in which the branch is introduced to the main chain should be carefully determined, as the introduction of a $\beta$-(1,6)-branch in the glycosyl donor and acceptor may affect $\beta$-(1,3)-glycosylation (Fig. 2 (c)).

As indicated above, the protecting groups and the leaving group that are introduced to the hydroxy groups have to be selected appropriately to enhance effective reactivity and stereoselectivity for glycosylation. Otherwise, synthesis becomes increasingly difficult. The study on the synthesis of $\beta$-(1,3)-glucan oligosaccharides is detailed below.

\section{C-1. Formation of the $\beta$ - $(1,3)$-Glucan Linkage}

To the best of my knowledge, the first synthesis of a $\beta-(1,3)$ bond was reported by Bächli et al. in 1952 (Fig. 3 (a)) (11). In that reports, $\beta$-(1,3)-glucan disaccharide 4 was synthesized from peracetylated glucosyl bromide $\mathbf{1}$ and diacetone glucose $\mathbf{2}$ used as a glycosyl donor and acceptor, respectively. Next, Klemer et al. achieved the introduction of a glucose residue at the 3- and 6-positions of the glucose acceptor in 1960 (12). For the synthesis of $\beta$-(1,3)-glycoside, they used a 4,6-O-benzylidene-protected accep- (a) 1. Electron-withdrawing effect by neighbored acyl groups

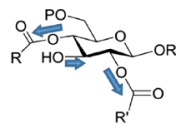

(b)

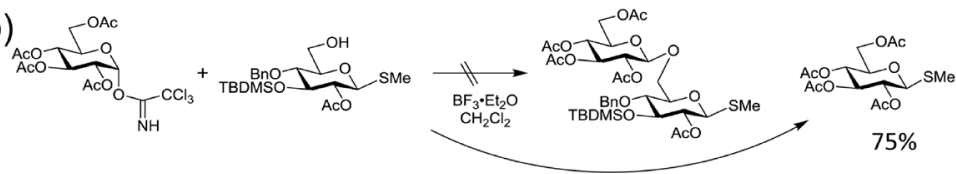

(c) 1. Steric hinderance by branched sugar

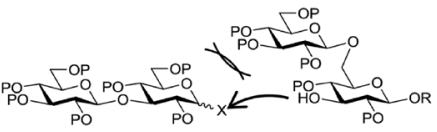

2. Steric hinderance around 3-position

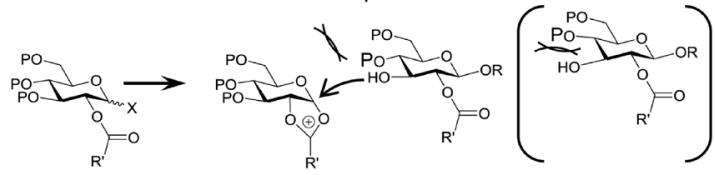

2. Partial glycosylation

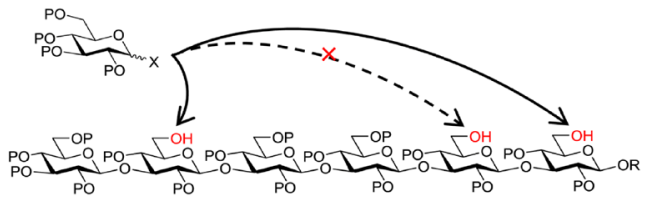

Fig. 2. (a) Challenge of $\beta$-(1,3)-bond formation induced by low reactive 3-hydroxy group; (b) aglycone transfer reaction using thioglycosides as glycosyl acceptor $(10)$; (c) challenge of branched $\beta$-(1,6)-bond formation. 


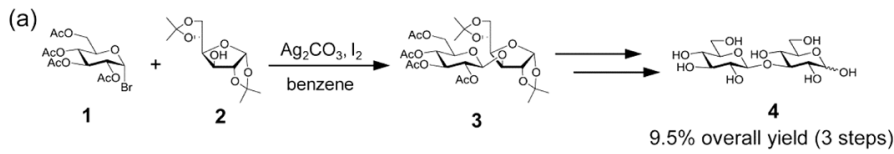

(b)

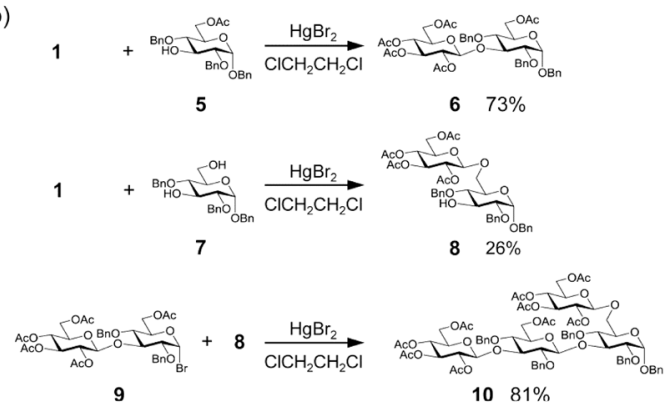

(c)
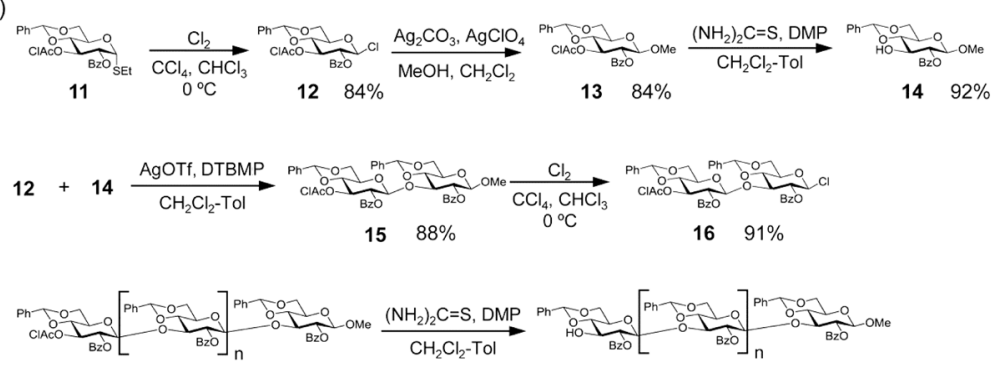

$\begin{array}{llll}15 n=0 & 17 n=0 & 91 \% \\ 18 n=2 & 19 & n=2 & 81 \% \\ 20 n=4 & 21 & n=4 & 80 \%\end{array}$

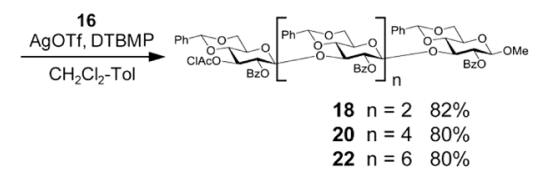

(d)
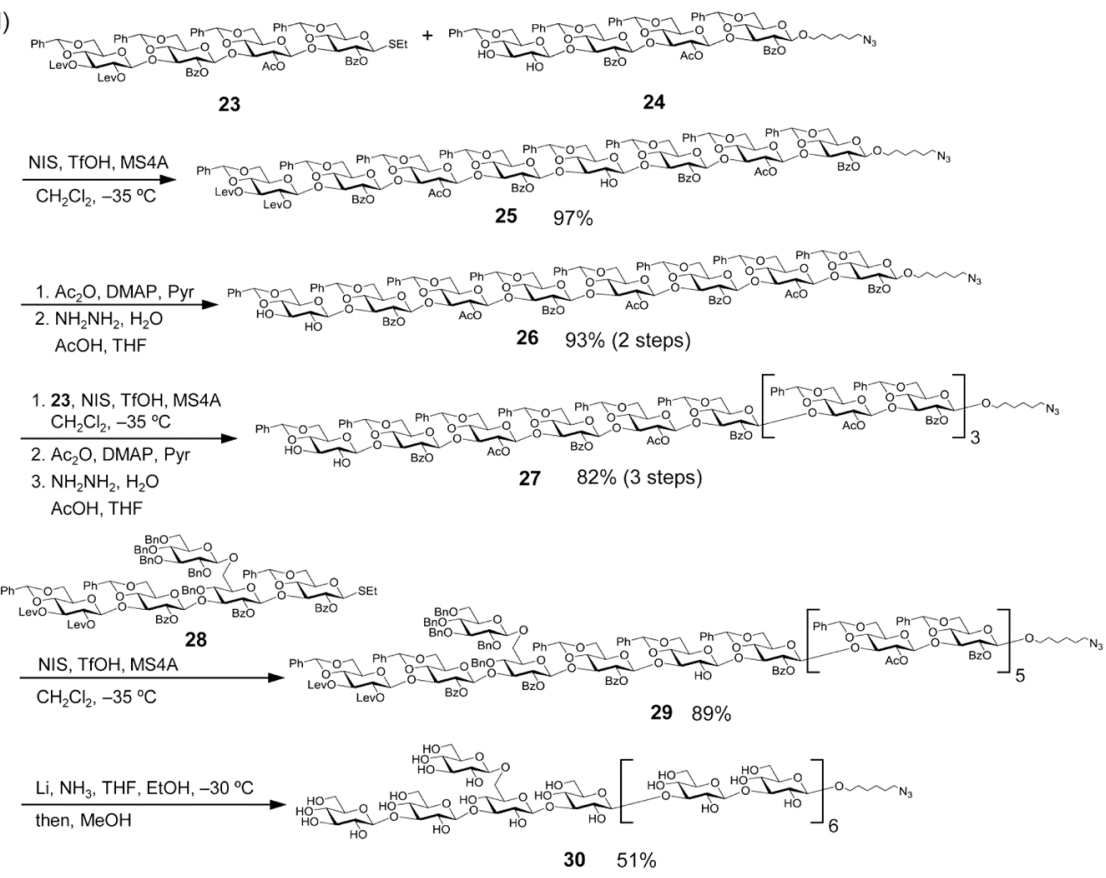

Fig. 3. (a) First $\beta$-(1,3)-bond formation reported by Bächli et al. (11); (b) synthesis of branched $\beta$-(1,3)-glucan tetrasaccharide by Ogawa et al. (14); (c) $\beta$-(1,3)-glucan oligosaccharide synthesis using disaccharide units by Takeo et al. (15); (d) synthesis of $\beta$-(1,3)-glucan dodecasaccharide using tetrasaccharide unit by Tanaka et al. (16). 
tor; however, non-regioselective glycosylation to the 2- and 3-position was a problem for use of this acceptor. In the following year, stepwise non-selective glycosylation was observed as a problem when the 2,3-diol acceptor was used; however, the product was the 3,6-glycosylated glucose (13).

In 1982, Ogawa et al. synthesized tetrasaccharide 10, which is composed of a main-chain trisaccharide with one glucose branch (Fig. 3 (b)). $\beta$-(1,3)-Disaccharide 6 was synthesized from 3-OH glucose acceptor 5 with peracetylated glucosyl bromide $\mathbf{1}$ in good yield. $\beta$-(1,6)-Disaccharide 8 obtained from 3,6-diol acceptor 7 was stepwisely glycosylated in high yield, with the $\beta$-(1,3)-disaccharyl bromide 9 converted from the $\beta$-(1,3)-disaccharide 6 to afford tetrasaccharide derivative $\mathbf{1 0}$ after conventional deprotection steps (14). Although this procedure is unique efficient method to achieve regioselective glycosylation, it seems not to be suitable as a forward-thinking plan for the synthesis of larger oligosaccharides, because the formation of a $\beta$-(1,3)-bond still remains difficult to produce in low yield. Additionally, due to harsh reaction conditions during the conversion to glycosyl bromide from the glycosylation product, the convergent synthesis of $\beta$-(1,3)-glucan using the glycosyl bromide was thought to be limited. Therefore, a thioglucoside as an alternative donor, which is stable and can be selectively activated, was introduced to enable the use of a variety of re-evaluated protecting groups, representing a breakthrough for the chemical synthesis of $\beta$-(1,3)-glucan.

\section{C-2. Synthesis of Linear $\beta-(1,3)-G l u c a n$ Oligosaccha- ride}

Optimization of the reaction conditions for the efficient formation of a $\beta$-(1,3)-bond has been the subject of intense research. This formation reaction must proceed in high yield as the repeatedly used key step to construct linear $\beta$-(1,3)-glucans. Multiple research teams are exploring many different pathways targeting this goal.

In 1993, Takeo et al. synthesized a linear octasaccharide 22 of $\beta$-(1,3)-glucan (Fig. 3 (c)) (15). Based on their previously reported approach to synthesis of $\beta$-glucan, disaccharide derivatives composed of 2-O-benzoyl- and 4,6-O-benzylidene-protected glucose residue with 3-O-chloroacetyl group in the non-reducing terminal residue were designed as a common intermediates $\mathbf{1 5}$. The thioglucoside $\mathbf{1 1}$ was converted to glycosyl chloride $\mathbf{1 2}$ as glycosyl donor, which was glycosidated with $\mathrm{MeOH}$ to give methyl glucoside $\mathbf{1 3}$. The 3-O-chloroacetyl group on the resulting glucoside $\mathbf{1 3}$ was deprotected efficiently to convert to glycosyl acceptor 14 . Then, glycosylation of glycosyl chloride 12 with glycosyl acceptor 14 gave $\beta$-(1,3)-disaccharide $\mathbf{1 5}$ as the common intermediate. Deprotection of 3-O-chloroacetyl group of the intermediate $\mathbf{1 5}$ was performed for conversion to the following glycosyl acceptor 17 . The glycosyl donor 16 was prepared by converting a 1-thioethyl group at the reducing end on the disaccharide derivative to chloride, which was efficiently glycosylated repeatedly with the acceptors 17, 19, 21 $(n=0,2,4)$ to achieve synthesis of octasaccharide 22 .

In 2010, chemical synthesis of $\beta$-(1,3)-glucan heptadecasaccharide $\mathbf{3 0}$ with the highest molecular weight was achieved by Tanaka et al. as shown in Fig. 3 (d) (16). The key element was that the glycosyl acceptor $\mathbf{2 4}$ protected the benzylidene groups at the 4,6-position of all residues, as well as an unprotected 2,3-OH in the non-reducing terminal residue. In the non-reducing terminal residue, the 4,6-cyclic protective group reduces steric hindrance at the 3 -position and, moreover, free 2,3-OH suppresses the decline in nucleophilicity of the $3-\mathrm{OH}$. The non-reducing end glucose of the glycosyl donor $\mathbf{2 3}$ has a levulinoyl group at the 2- and 3-positions. After glycosylation, the levulinoyl groups of the derivative were deprotected to afford a glycosyl acceptor 26 with free 2,3-OH. Using a tetrasaccharide unit $\mathbf{2 3}$ as a connection block, the elongation of glycan chain proceeded efficiently to afford the hexadecasaccharide. As the last block, a branched heptasaccharide $\mathbf{2 8}$ that was prepared separately was introduced to the dodecasaccharide acceptor $\mathbf{2 7}$ to give a branched heptadecasaccharide 29. It is worth noting that the glycosylation step using the tetrasaccharide unit $\mathbf{2 3}$ was more efficient than that for preparation of the tetrasaccharide unit using disaccharide units. Additionally, deprotection of all protecting groups on synthetic $\beta$-(1,3)-glucan can be completed in onepot by birch reduction and saponification during quenching of the reduction.

Kong's group used highly $O$-acylated glycosyl donors for the synthesis (Fig. 4 (a)-1). In their research, glycosyl imidates were examined mainly with acetyl groups except using an allyl group at the 3-position of the non-reducing residue for further elongation. Glycosylation reactions with the imidates $\mathbf{3 1}$ and $\mathbf{3 4}$ are $\alpha$-selective with no apparent $\beta$-selectivity by neighboring-groups (17). However, glycosylation using 2,3,4,6-tetra- $O$-benzoylglucosyl imidate 39 in which only the benzoyl group was used as a protecting group provided the corresponding $\beta$-product $\mathbf{4 0}$ more selectively than the corresponding tetra- $O$-acetylated donor 37 (43, $\alpha: \beta=1: 2$ ). Zeng et al. proposed that the formation of $\alpha$-glucoside may proceed via orthoester formation followed by $\beta$-triflate generation with trimethylsilyl trifluoromethanesulfonate (TMSOTf) and transfer of aglycone from the $\alpha$-face (Fig. 4 (a)-2). 2-O-Acetyltype disaccaharyl imidates were examined as shown in Fig. 4 (b). The glycosylation of glucosyl- $\alpha-(1,3)$-glucosyl imidate 41 with glucosyl- $\alpha$-(1,3)-glucosyl acceptor 42 was shown to be $\beta$-selective whereas that of glucosyl- $\beta$-(1,3)-glucosyl imidate 44 with glucosyl- $\beta$-(1,3)-glucosyl acceptor 45 was $\alpha$-selective (18). These 
(a) 1. Glycosylation

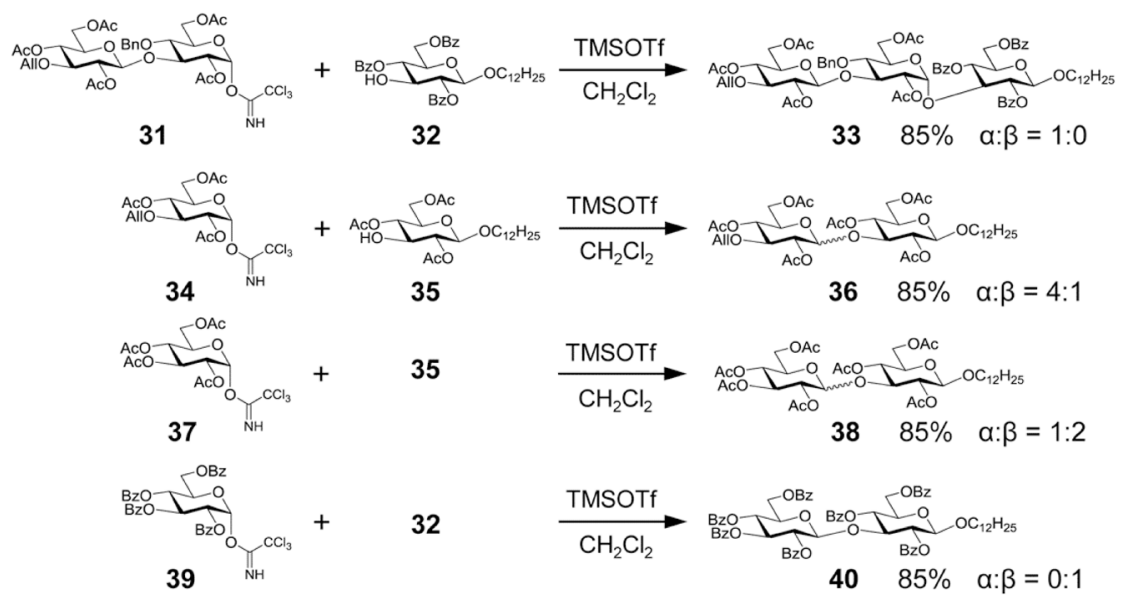

2. Predicted mechanism of the a-selective glycosylation

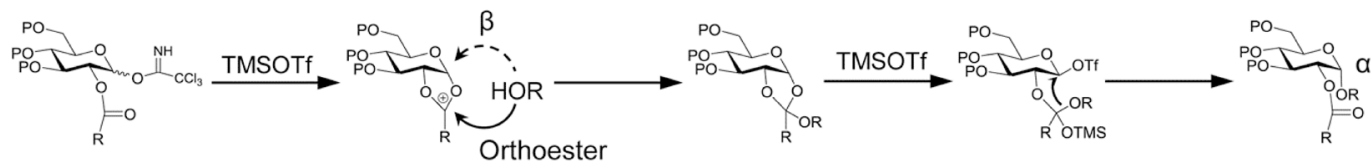

(b)

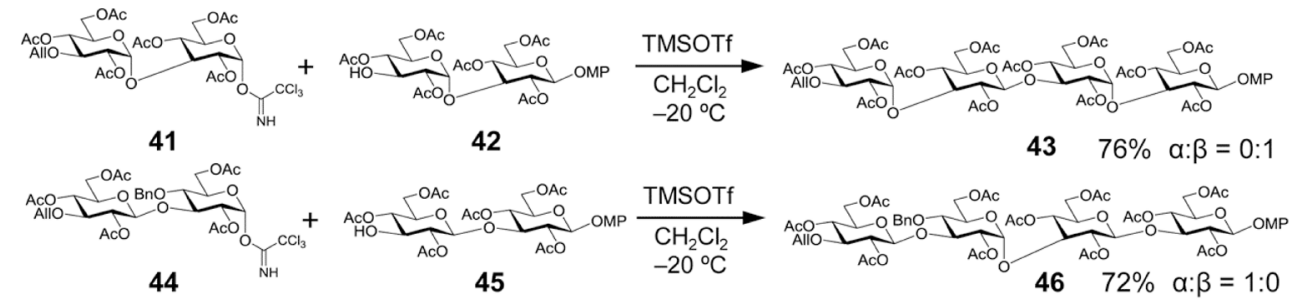

Fig. 4. (a) Challenge of stereoselectivity in glycosylation using highly acylated substrates reported by Zeng et al. (17); (b) substrate structure dependent stereoselectivity in $\boldsymbol{\beta}$-(1,3)-glycosylation (18).

results indicate that not only neighboring group participation, but also structures further away from the glycosylating point rather influence the stereoselectivity of glycosylation strongly. These unpredictable results complicated the establishment of a method for providing $\beta$-(1,3)-bonds, which was deeply recognized to be difficult. Upon receiving Kong's reports, Ensley's group introduced a 4-acetoxy-2,2-dimethylbutanoyl (ADMB) group to the 2-position of the glycosyl donor 47 and the $\beta$-(1,3)-glycosylation reaction produced high yield and high selectivity (Fig. 5 (a)) $(19,20)$. It was considered that the ADMB group sterically prevented from orthoester formation with neighboring group participation, efficiently controlling $\beta$-selectivity.

Weishaupt et al. attempted to synthesize long $\beta$-(1,3)-glucan oligosaccharide by continuous glycosylation with solid phase synthesis (21). They considered that benzylidene group on the glucose residue 52 in combination with the bulky pivaloyl ester protecting group diminished reactivity of 3-OH in the glycosylation reaction (Fig. 5 (b)-1). Therefore, using a glycosyl donor 55 whose rigid 4,6-O-benzylidene group was substituted with flexible two benzyl groups and whose 5-tert-butyl-2-methylbenzenethiol was replaced by more reactive dibutyl phosphate, solid-phase synthesis of linear dodecasaccharide 57 was accomplished (Fig. 5 (b)-2). The average yield of each reaction and overall yield were $88.4 \%$ and $4.6 \%$, respectively, with 25 steps in the reaction including glycosylations and deprotections. Because benzyl groups are more stable than a benzylidene group during solid phase synthesis, which repeatedly requires the use of a large quantity of Lewis acid with glycosyl donor for completion of each glycosylation, the glycosylation yield using benzyl ethers in the glucose residue was surmised to be improved.

To provide $\beta$-selective glycosylation for synthesis of $\beta$-(1,3)-glucan oligosaccharides, the 4,6-O-benzylidene-protected glucosyl acceptor is thought to be effective and is the first choice except for special cases such as solid-phase synthesis. Because the cyclic protective group of 4,6-diol strongly ties the 4-O group in the direction of the 6-O group, the 4,6-O-benzylidene group reduces the steric hindrance around the $3-\mathrm{OH}$ of the acceptor, which leads to a high yield of $\beta$-selective glycosylation. Recently, Liao 
(a)
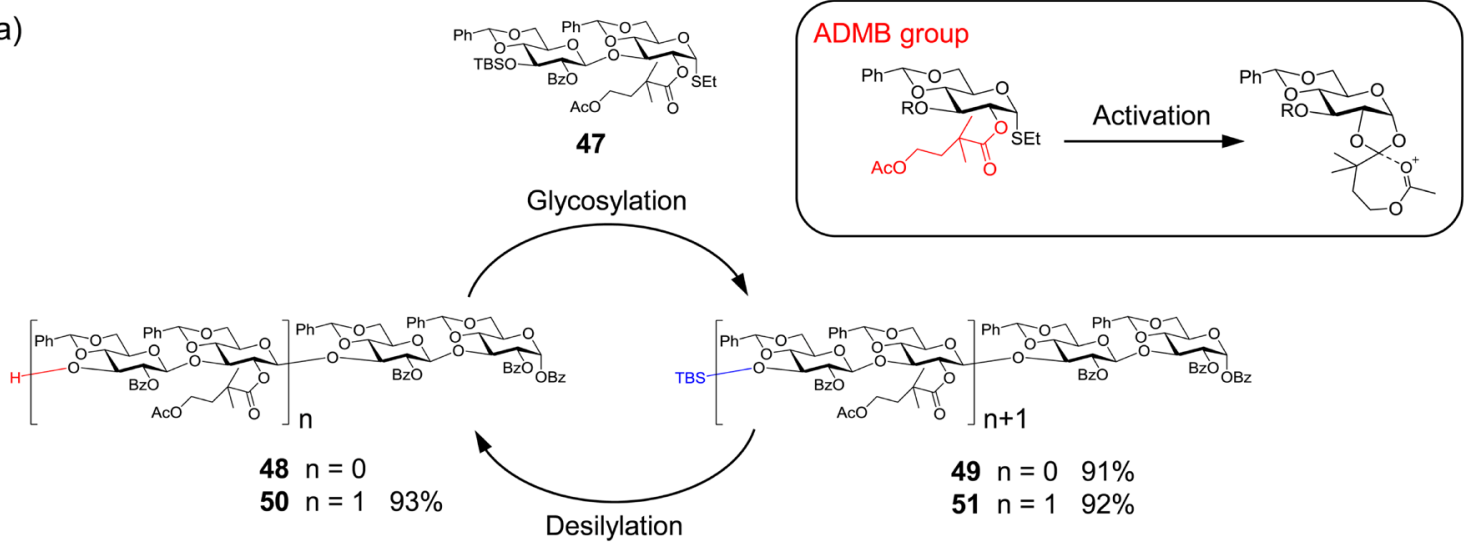

(b) 1 .

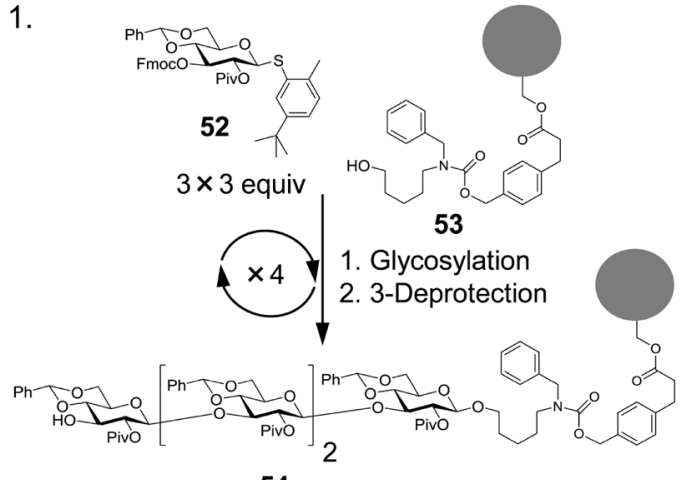

2.

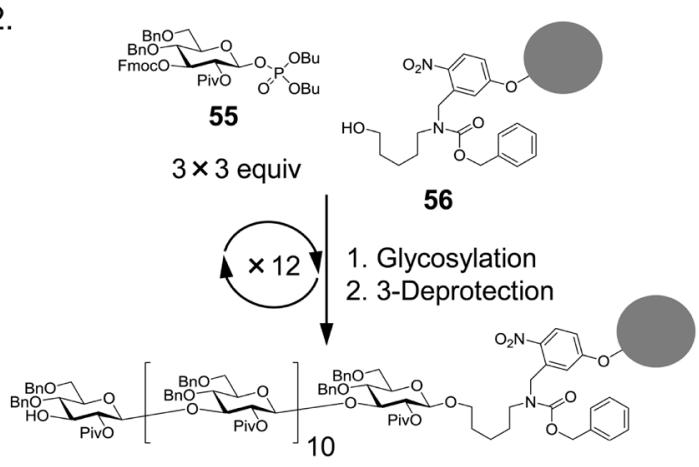

57

Fig. 5. (a) Synthesis of $\beta$-(1,3)-glucan oligosaccharide using neighboring participation by ADMB group (19, 20); (b) solid-phase synthesis of $\boldsymbol{\beta}$-(1,3)-glucan dodecasaccharide (21).

et al. synthesized linear $\beta$-(1,3)-glucan dodecasaccharide using a common benzylidenated intermediate with a 2-O-benzoylated structure that can be converted to both glycosyl donor and acceptor with similar protective groups (22). This confirmed the efficacy of the benzylidene group to synthesize linear $\beta$-(1,3)-glucan oligosaccharide. However, it is important to further consider that alternative protecting groups are necessary for introduction of the branching residue.

\section{C-3. Synthesis of Branched $\beta-(1,3)$-Glucan Oligosac- charide}

Introduction of $\beta$ - $(1,6)$-branch chains has not been thoroughly investigated because the construction of backbone $\beta$-(1,3)-chain had been the focus until recently as mentioned above. Although the formation of the $\beta$-(1,6)-bond requires less problematic glycosylation with a highly reactive primary hydroxy group, the synthetic strategy considering not only the structure of the acceptor for the introduction of the branched structure, but also when to assemble the branched structure should be designed. The structure of the glycosyl acceptor and donor designed for the introduction of the branched structure may influence $\beta$-(1,3)-bond formation (Fig. 2 (c)).
In 2000, Du et al. glycosylated the reducing end of the $\beta$-(1,3)-backbone residue of $\beta$-(1,3)- and (1,6)-linked trisaccharide $\mathbf{6 2}$ using thioglycoside of a similar trisaccharide $\mathbf{6 4}$ to produce a branched $\beta$-(1,3)-glucan hexasaccharide $\mathbf{6 5}$ in 50\% yield (Fig. 6 (a)) (23). Although the yield was low due to the steric hindrance of the branch, $\beta$-selectivity was maintained. In 2002, He et al. attempted to glycosylate a $\beta$ - $(1,6)$-linked disaccharide acceptor $\mathbf{7 0}$ with trisaccharide donor $\mathbf{6 6}$ with the branch at the reducing end, but did not succeed (Fig. 6 (b)) (24). However, glycosylation with a 4,6-O-benzylidenated 2,3-diol acceptor $\mathbf{6 7}$ proceeded efficiently. Therefore, the use of a glycosyl acceptor with a branch on the glycosylated residue decreases the yield. In a following study (Fig. 6 (c)) (25), trisaccharide imidate $\mathbf{7 0}$ was used as a glycosyl donor to introduce the branched trisaccharide acceptor $\mathbf{7 1}$ at the nonreducing end residue of the $\beta$-(1,3)-main chain. However, in the case of this branched trisaccharide imidate 70, $\alpha$-selective glycosylation was also observed, similar to Zeng's result shown in Fig. 4 (b). Considering these reports, it should be noted that structures with acyl groups at many positions produce unanticipated selectivity in glycosylation.

Tanaka et al. reported an attractive method for synthesis of 
(a)
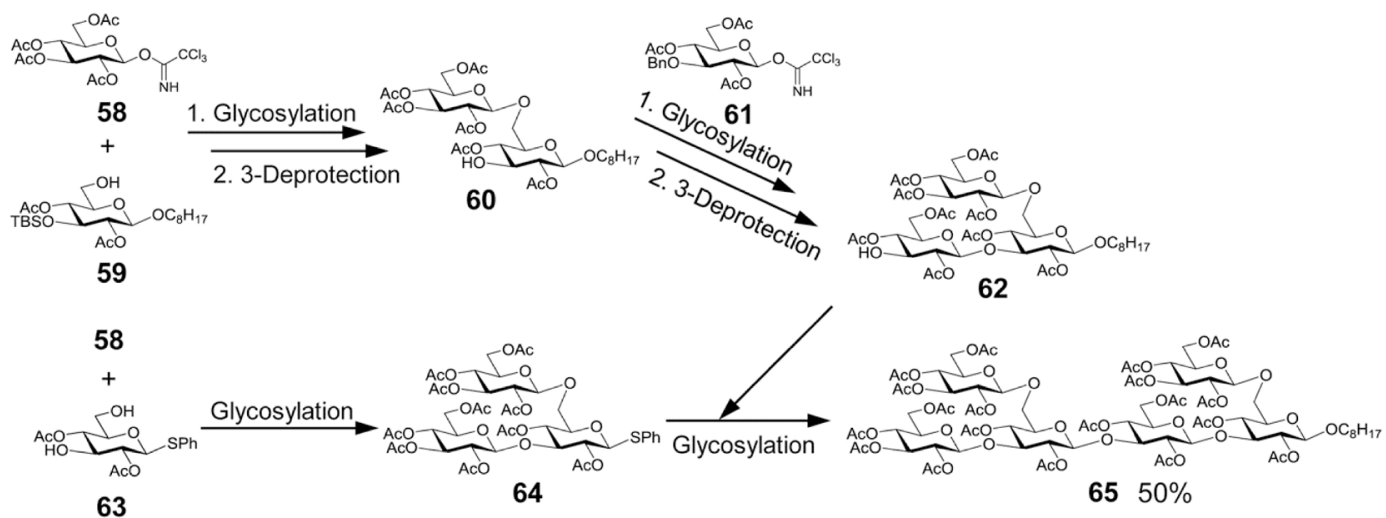

(b)

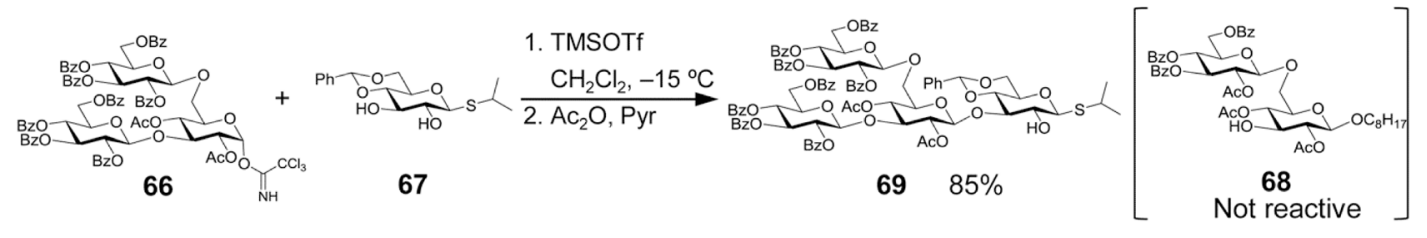

(c)

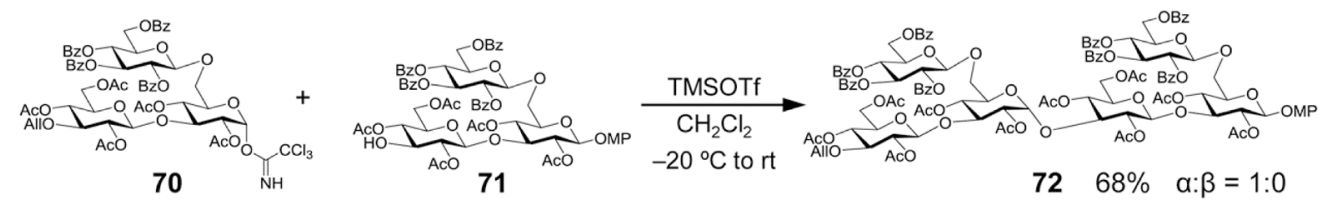

Fig. 6. (a) Synthesis of branched $\beta$-(1,3)-glucan hexasaccharide (23); (b) influence of branched unit for $\beta$-(1,3)-glycosylation (24); (c) observed $\alpha$-selective glycosylation using $2-O$-acetylated trichloroacetimidate (25).

branched $\beta$-(1,3)-glucan oligosaccharide (Fig. 7 (a)) (26). In the synthesis of linear tetrasaccharide $\mathbf{7 4}$, different protecting groups at the 6-position of each glucose residue were introduced. This suggested that a branching residue could be selectivity introduced to the tetrasaccharide structure. The branch was introduced selectively to the glucose residue whose Fmoc group, Lev group, or Alloc group was deprotected. This method to produce branches at different positions could be efficiently used to clarify the biological importance of the branch in $\beta$-(1,3)-glucan. This introduction method of the branch enables to control of complete synthesis based on synthetically chemical design.

Next, the $\beta$-(1,3)-linked disaccharide derivative $\mathbf{7 8}$ and $\beta$-(1,6)-linked disaccharide derivative $\mathbf{8 1}$ were converted to glycosyl donors (79 and $\mathbf{8 2}$ ) and glycosyl acceptors (80 and $\mathbf{8 3})$ to study the effect of the branch on the glycosyl donor or the glycosyl acceptor in $\beta$-(1,3)-glycosylation (Fig. 7 (b)) (10). The $\beta$-(1,6)-linked disaccharide donor $\mathbf{8 2}$ glycosylated the $\beta$ - $(1,3)$-linked disaccharide acceptor 80 to give the tetrasaccharide 85 in a $56 \%$ yield, whereas the $\beta$-(1,6)-linked disaccharide acceptor $\mathbf{8 3}$ was glycosylated with the $\beta$-(1,3)-linked disaccharide donor $\mathbf{7 9}$ to give the tetrasaccharide $\mathbf{8 4}$ in a $15 \%$ yield. These results were caused by steric hindrance of the branch. The branch on the glycosyl acceptor hindered $\beta$-(1,3)-glycosylation more sterically than that on the glycosyl donor. This result suggested that glycosylation at the 3-position of the branching glucose residue should be avoided. Then, a branching glucose residue was introduced at the 6-position of the second residue in the linear $\beta$-(1,3)-linked trisaccharide $\mathbf{8 7}$ as the repeating branched tetrasaccharide unit of schizophyllan. This repeating unit 90 is less steric and more reactive at both non-reducing and reducing terminal non-branched glucose residues. It was thus suggested that the repeating unit $\mathbf{8 8}$ can be used as both a glycosyl acceptor and donor for construction of branched $\beta$ - $(1,3)$-glucan polysaccharides.

Liao et al. achieved synthesis of $\beta$-(1,3)-glucan oligosaccharide with a long branch chain (Fig. 8) (27). First, tetrasaccharide blocks 89 and 94 were synthesized by three continuous glycosylations in a one-pot reaction. To obtain the branched structure, a linker monosaccharide unit $\mathbf{9 0}$ obtained through regioselective ring-opening from a 4,6-O-benzylidene-protected unit was used. The connection of three tetrasaccharide blocks, 89, 91, and 94, proceeded efficiently at the linker unit $\mathbf{9 0}$ to produce a branched tridecasaccharide 95 through a linear nonasaccahride $\mathbf{9 3}$.

Although there have been fewer reports of branched $\beta$-(1,3)-glucan oligosaccharide synthesis than those of linear 
(a)
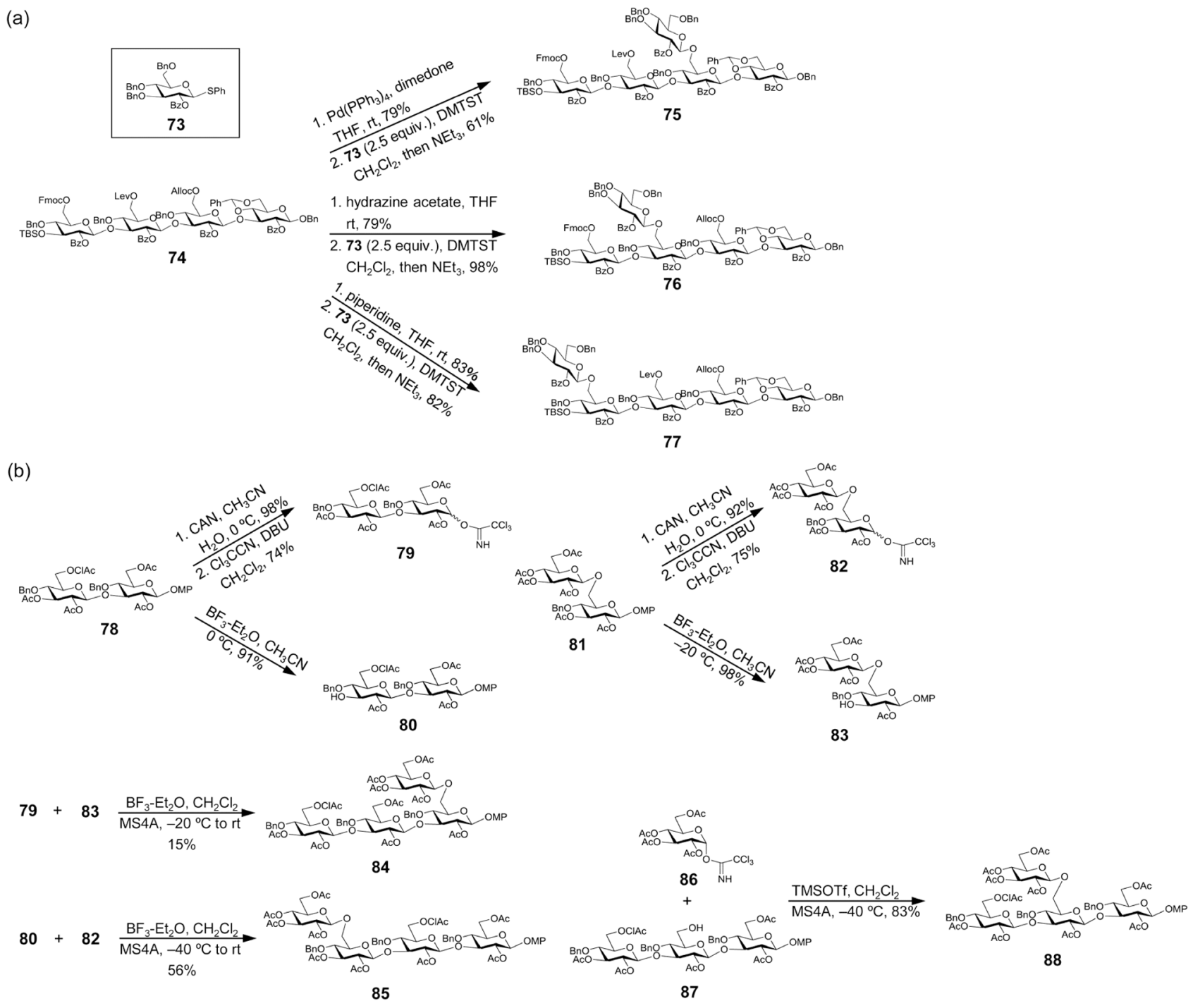

77

Fig. 7. (a) Regioselective introduction of branch at 6-position by orthogonal deprotection and glycosylation (26); (b) effect of a position of branched $\beta-(1,6)$ residue in chemical conversion and glycosylation $(10)$.

$\beta$-(1,3)-glucan oligosaccharide synthesis to date, introduction of pre-constructed branch residues after construction of the preconstructed linear backbone as the very last stage in the synthesis of branched $\beta$-(1,3)-glucan oligosaccharides has been discovered to proceed effectively. Similar to what Liao et al. reported previously (27), we also found that units with a branching interior residue in the linear $\beta$-(1,3)-backbone and a non-branched residue at both the non-reducing and reducing terminals could be used for alternative effective construction of a repeating branched $\beta$-(1,3)-glucan structure. Our results also support the validity of these strategy (unpublished data).

\section{Application of Chemical Synthetic $\beta-(1,3)$-Glucan Oligosaccharide}

In recent years, it has become possible to synthesize $\beta$-(1,3)-glucan oligosaccharides as mentioned before. The synthetic $\beta$-(1,3)-glucan oligosaccharides enable detailed biological analysis with a defined homogeneous structure.

In 2005, Jamois et al. synthesized a linear pentasaccharide and analyzed its structure using NMR. Moreover, the tetrasaccharide and pentasaccharide were used for comparison with phycarine (linear $\beta$-(1,3)-glucan, molecular weight of approximately 5000) regarding immunological activity (28). All three molecules similarly stimulated macrophage phagocytic activity. Moreover, the two oligosaccharides induced the cytokine IL- $1 \beta$ and inhibited tumor cell growth with a potency similar to that of phycarine. This result for low-molecular-weight $\beta$-(1,3)-glucans suggested that triple helix conformation of $\beta$-(1,3)-glucan is not always necessary to stimulate an immune response. This result clearly promotes the subsequent inspection of biological activity of synthetic $\beta$-(1,3)-glucan, 


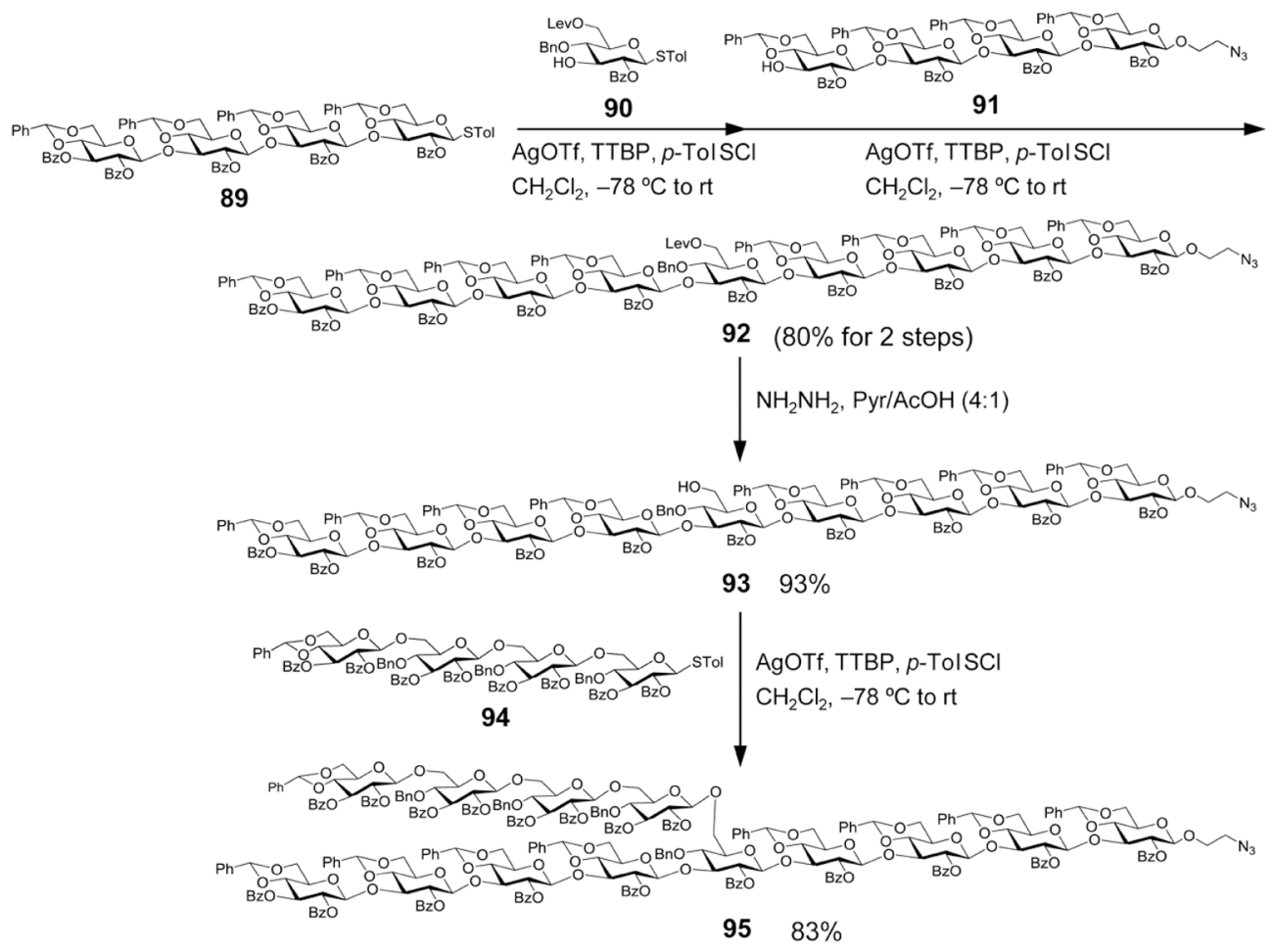

Fig. 8. Synthesis of $\beta$-(1,3)-glucan tridecasaccharide with a long branch (27).

which is free from biological impurities (e.g., proteins, lipids, and carbohydrates).

Adams et al. and Brown et al. analyzed the interactions of Dectin-1 with natural $\beta$-(1,3)-glucan and synthetic $\beta$-(1,3)-glucan oligosaccharide (29). In that study, tens of thousands of molecular weights of $\beta$-(1,3)-glucan were used for competitive binding experiments to Dectin-1. The results indicated that oligosaccharides with heptasaccharide to decasaccharide lengths inhibited binding of natural $\beta$-(1,3)-glucan in the order of $\mathrm{mM}$. Moreover, decasaccharide with one branched glucose residue showed 100-fold stronger affinity than a linear decasaccharide. In contrast, a commercially available naturally occurring branched $\beta$ - $(1,3)$-glucan, laminarin with a molecular weight of 7700 showed inhibitory activity at $22 \mathrm{nM}$. Therefore, the active structure of synthetic $\beta$-(1,3)-glucan oligosaccharide binding to Dectin-1 should have a suitable length with similar molecular weight to the decasaccharide and proper branching.

A recent notable study from Tanaka et al. performed a competitive inhibition assay for the binding of schizophyllan and deca- through hexadecasaccharides to Dectin-1 (30). The linear hexadecasaccharide and branched heptadecasaccharide had onetenth the activity of schizophyllan, and inhibited binding strongly. However, for induction of NF- $\kappa \mathrm{B}$, they had one-hundredth of the activity. Furthermore, the binding position of the linear hexadecasaccharide in the Dectin-1 binding domain was determined using a saturation transfer difference (STD)-NMR technique. The signals of hydrogens at the 1,3-positions on the inner glucose provided high STD-amplification factors $(100 \%$ and $87 \%)$ and that at the 2,4,6-positions showed the moderate ones (72-77\%). However, the alkyl chain of the aglycone and the glucose of the reducing end and the non-reducing end produced weak signals $(8-15 \%)$. Therefore, it was concluded that Dectin-1 recognizes inner glucose residues. Considering previous reports that confirm the physiological activity of natural $\beta$-(1,3)-glucans, it is necessary to perform further glycosylation to synthesize $\beta$-(1,3)-glucan oligosaccharide with a higher molecular weight.

A vaccine that covalently binds synthetic $\beta$ - $(1,3)$-glucans to carrier proteins has been developed. Donadei et al. reported the multiple binding of synthesized linear $\beta$ - $(1,3)$-glucan hexasaccharide to detoxified diphtheria toxin $\left(\mathrm{CRM}_{197}\right)(31)$. Evaluation of the binding affinity with Dectin-1 and cell viability revealed that $\mathrm{CRM}_{197}$ bound $18.2 \beta$-(1,3)-glucan hexasaccharides had immunological activity similar to that of the conjugate bound with naturally occurring linear $\beta$-(1,3)-glucan, curdlan. Additionally, $\mathrm{CRM}_{197^{-}}$ specific antibody titers were analyzed in mice. $\mathrm{CRM}_{197}$ conjugated with synthetic $\beta$-(1,3)-glucan oligosaccharide elicited significantly higher production of $\mathrm{IgG}$ antibody than $\mathrm{CRM}_{197}$ alone or the conjugate with curdlan. These results indicate that the $\beta$ - $(1,3)$-glucan hexasaccharide is a useful antibody adjuvant for development of vaccine-conjugated $\beta$-(1,3)-glucan. Next, Liao et al. developed a 
(a)

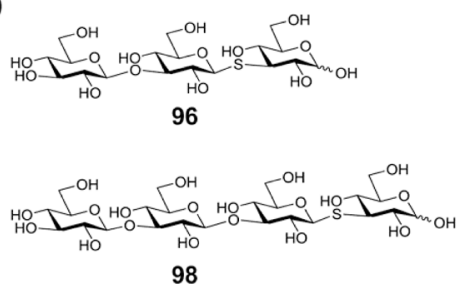

(b)

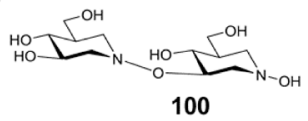

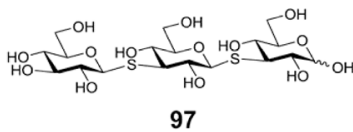
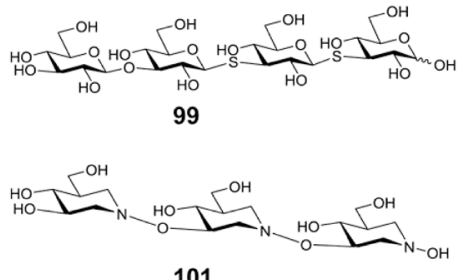

101

Fig. 9. (a) $\beta$-(1,3)-Glucan mimics having $S$-glycosides (33); (b) $\beta$-(1,3)-glucan mimics linked with $\mathbf{N}-\mathbf{O}$ bonds (34).

$\beta$-(1,3)-glucan-specific vaccine (32). $\beta$-(1,3)-Glucan linear nonasaccharides with different lengths of branch chains were conjugated covalently with keyhole limpet hemocyanin (KLH). These conjugates were injected subcutaneously into mice and the antibody manufacturability and specificity were evaluated. The production of IgG and IgM antibodies was found to occur in high levels and the specificity of these antibodies was high against the $\beta$-(1,3)-glucan moiety used in the conjugate. These results indicate a possibility to develop a vaccine specific for the $\beta$-(1,3)-glucan structure in fungal cell walls.

As effective synthetic pathways for $\beta$-(1,3)-glucan have been recently developed, further synthesis of $\beta$ - $(1,3)$-glucan mimics has been also reported. Sylla et al. synthesized a $\beta$-(1,3)-glucan oligosaccharide with $S$-glycoside 96-99 as the linkage rather than the natural $O$-glycoside linkage (Fig. 9 (a)) (33); these molecules stimulated macrophage phagocytosis dependent on the length of the $\beta$-(1,3)-glucan mimic. Cytokine production induced by the $\beta$-(1,3)-glucan mimic was similarly observed. Additionally, the synthetic mimics were evaluated for suppression of cancerization and metastasis using tumor stem cells. Surprisingly, the shorter trisaccharide mimic $\mathbf{9 7}$ having only the $S$-linked structure showed suppression activity similar to that of natural laminarin with high molecular weight. Additionally, Ferry et al. synthesized $\beta$-(1,3)-glucan mimics $\mathbf{1 0 0}$ and $\mathbf{1 0 1}$ that were oligopiperidines linked with hydroxylamine (Fig. 9 (b)) (34), which resulted in a more hydrophobic $\alpha$-face than $\beta$-(1,3)-glucan and an achiral $\mathrm{N}-\mathrm{O}$ bond rather than $\beta$-O-glycosidic bonds. Although oligopiperidine $\beta$-(1,3)-glucan mimics have a low molecular weight, these mimics inhibited anti-CR3 and anti-Dectin-1 antibody binding to neutrophils and macrophages. Moreover, they also stimulated phagocytosis by macrophages. Since these mimics bound to $\beta$ - $(1,3)$-glucan recognition sites and had $\beta$-(1,3)-glucan-like activity, further study on $\beta$-glucan mimics leads a novel development in small molecule therapeutics through $\beta$-(1,3)-glucan pathway.

Weishaupt et al. completed the deprotection of protected $\beta$-(1,3)-glucan tridecasaccharide overnight by substitution of 2-Opivaloyl groups with 2-O-benzoyl groups on the glycosyl donor to improve their solid-phase $\beta$-(1,3)-glucan oligosaccharide synthesis reported previously in 2013 (21), because it took 2 weeks to deprotect the 2-O-pivaloyl groups completely (35). Additionally, a 6-O-levulinoyl protected glycosyl donor was used efficiently in the improved solid-phase $\beta$-(1,3)-glucan oligosaccharide synthesis toward branched $\beta$-(1,3)-glucan structures. The branched $\beta$-(1,3)-glucan oligosaccharides with different lengths of the linear $\beta$-(1,3)-chain were immobilized on a microarray that was utilized for analysis of the anti- $\beta$-(1,3)-glucan IgG antibody. The results of the microarray assay of human serum supported the production of an anti- $\beta$-(1,3)-glucan antibody against both linear and branched structures. The $\beta$-(1,3)-glucan-immobilized microarray may also serve as a valuable tool to analyze the exact antigen specificity of anti- $\beta$-(1,3)-glucan antibodies.

As discussed above, an efficient method for chemical synthesis of $\beta$-(1,3)-glucan oligosaccharide has continued to be established, step-by-step, in recent years. The synthesis of $\beta$-(1,3)-glucan oligosaccharide allows the preparation of homogeneitycontrolled $\beta$-(1,3)-glucan samples, which in turn enables precise biological study. Synthetic $\beta$-(1,3)-glucan oligosaccharides have been shown to have similar activity to natural $\beta$ - $(1,3)$-glucan when the length of $\beta$-(1,3)-glucan oligosaccharide can approximate the active ligand structure for the receptor protein. To date, synthesis of $\beta$-(1,3)-glucan up to approximately decasaccharide length has become definitively possible, but this length is still not long enough to mimic the active fragment of natural $\beta$-(1,3)-glucans. The recent progresses in chemical synthesis of $\beta$ - $(1,3)$-glucan oligosaccharide suggest that total synthesis of $\beta$ - $(1,3)$-glucan polysaccharides and the development of new drugs showing equivalent or higher immunological activity compared to native $\beta$-( $(1,3)$-glucan might become available the near feature. 


\section{References}

1. Thomas, S., Durand, D., Chassenieux, C., and Jyotishkumar, P. (2013) Handbook of Biopolymer-Based Materials: From Blends and Composites to Gels and Complex Networks, Wiley-VCH Verlag GmbH \& Co. KGaA.

2. McIntosh, M., Stone, B. A., and Stanisich, V. A. (2005) Appl. Microbiol. Biotechnol. 68, 163-173.

3. Tsvetkov, Y. E., Khatuntseva, E. A., Yashunsky, D. V., and Nifantiev, N. E. (2015) Russ. Chem. Bull. 64, 990-1013.

4. Ren, L., Perera, C., and Hemar, Y. (2012) Food Funct. 3, 1118-1130.

5. Chan, G. C. F., Chan, W. K., and Sze, D. M. Y. (2009) J. Hematol. Oncol. $2,25$.

6. Brown, G. D., and Gordon, S. (2001) Nature 413, 36-37.

7. Brown, G. D., Herre, J., Williams, D. L., Willment, J. A., Marshall, A. S., and Gordon, S. (2003) J. Exp. Med. 197, $1119-1124$.

8. Legentil, L., Paris, F., Ballet, C., Trouvelot, S., Daire, X., Vetvicka, V., and Ferrières, V. (2015) Molecules 20, $9745-9766$.

9. Adachi, Y. (2007) Trends Glycosci. Glycotechnol. 19, 195-207.

10. Miyagawa, A., Matsuda, T., and Yamamura, H. (2015) J. Carbohydr. Chem. 34, 215-246.

11. Bächli, P., and Percival, E. G. V. (1952) J. Chem. Soc. 0, 1243-1246.

12. Klemer, A., and Homberg, K. (1960) Chem. Ber. 93, 1643-1649.

13. Klemer, A., and Homberg, K. (1961) Chem. Ber. 94, 2747-2754.

14. Ogawa, T., and Kasuragi, T. (1982) Carbohydr. Res. 103, 53-64.

15. Takeo, K., Maki, K., Wada, Y., and Kitamura, S. (1993) Carbohydr. Res. 245, 81-96.

16. Tanaka, H., Kawai, T., Adachi, Y., Ohno, N., and Takahashi, T. (2010) Chem. Commun. (Camb.) 46, 8249-8251.

17. Zeng, Y., Ning, J., and Kong, F. (2002) Tetrahedron Lett. 43, 3729-3733.

18. Zeng, Y., Ning, J., and Kong, F. (2003) Carbohydr. Res. 338, 307-311.

19. Yu, H., Williams, D. L., and Ensley, H. E. (2005) Tetrahedron Lett. 46, 3417-3421.

20. Mo, K.-F., Li, H., Mague, J. T., and Ensley, H. E. (2009) Carbohydr. Res. 344, 439-447.

21. Weishaupt, M. W., Matthies, S., and Seeberger, P. H. (2013) Chemistry 19, 12497-12503.

22. Liao, G., Zhou, Z., Burgula, S., Liao, J., Yuan, C., Wu, Q., and Guo, Z. (2015) Bioconjug. Chem. 26, 466-476.

23. Du, Y., Zhang, M., and Kong, F. (2000) Org. Lett. 2, 3797-3800.

24. He, H., Yang, F., and Du, Y. (2002) Carbohydr. Res. 337, 1673-1678.

25. Zicheng, W., Ning, J., and Kong, F. (2003) Carbohydr. Res. 338, 2203-2212.

26. Tanaka, H., Amaya, T., and Takahashi, T. (2003) Tetrahedron Lett. 44, 3053-3057.

27. Liao, G., Burgula, S., Zhou, Z., and Guo, Z. (2015) Eur. J. Org. Chem. 2015, 2942-2951.

28. Jamois, F., Ferrieres, V., Gue gan, J., Yvin, J., Plusquellec, D., and Vetvicka, V. (2005) Glycobiology 15, $393-407$.

29. Adams, E. L., Rice, P. J., Graves, B., Ensley, H. E., Yu, H., Brown, G. D., Gordon, S., Monteiro, M. A., Papp-Szabo, E., Lowman, D. W., Power, T. D., Wempe, M. F., and Williams, D. L. (2008) J. Pharmacol. Exp. Ther. 325, 115-123.

30. Tanaka, H., Kawai, T., Adachi, Y., Hanashima, S., Yamaguchi, Y., Ohno, N., and Takahashi, T. (2012) Bioorg. Med. Chem. 20, $3898-3914$.

31. Donadei, A., Gallorini, S., Berti, F., O’Hagan, D. T., Adamo, R., and Baudner, B. C. (2015) Mol. Pharm. 12, $1662-1672$.

32. Liao, G., Zhou, Z., Liao, J., Zu, L., Wu, Q., and Guo, Z. (2015) ACS Infect. Dis. 2, 123-131.

33. Sylla, B., Legentil, L., Saraswat-Ohri, S., Vashishta, A., Daniellou, R., Wang, H. W., Vetvicka, V., and Ferrières, V. (2014) J. Med. Chem. 57, $8280-8292$.

34. Ferry, A., Malik, G., Guinchard, X., Větvička, V., and Crich, D. (2014) J. Am. Chem. Soc. 136, 14852-14857.

35. Weishaupt, M. W., Hahm, H. S., Geissner, A., and Seeberger, P. H. (2017) Chem. Commun. (Camb.) 53, 3591-3594.

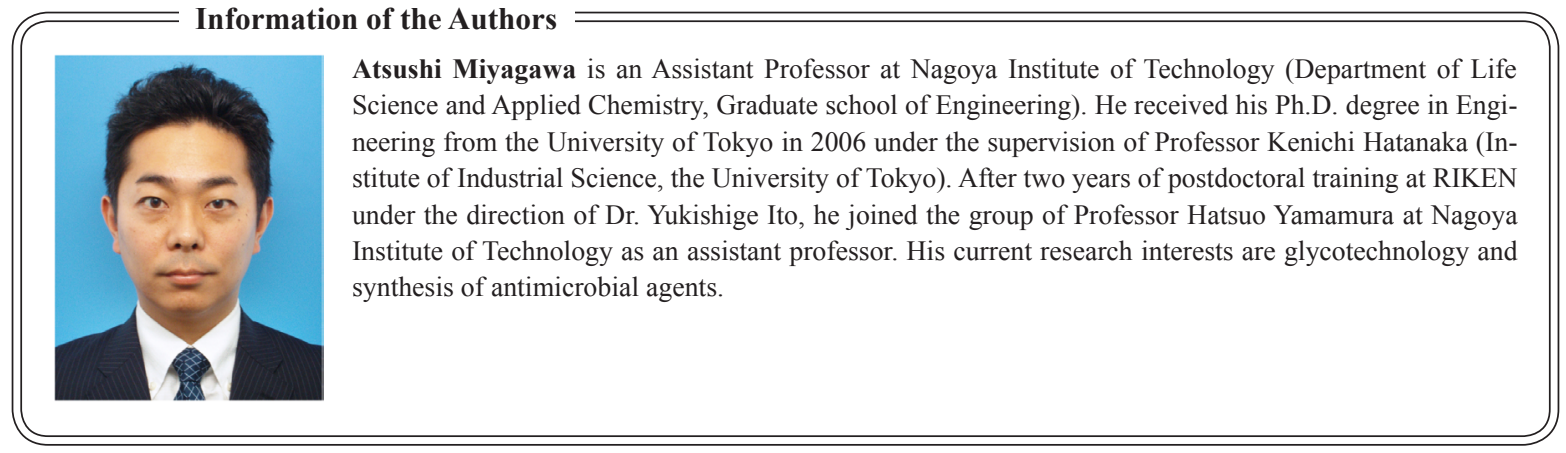

